\title{
EL DESARROLLO DE COMPETENCIAS GENÉRICAS EN LA EDUCACIÓN TÉCNICA DE NIVEL SUPERIOR: UN ESTUDIO DE CASO
}

\author{
Gerald Pugh ${ }^{1}$ \\ Armando Lozano-Rodríguez ${ }^{2}$
}

\begin{abstract}
RESUMEN
Este estudio pretende mejorar la comprensión del proceso de desarrollo de competencias genéricas en estudiantes de carreras técnicas de nivel superior, mediante la percepción de los propios estudiantes. Por medio de un estudio cualitativo de caso de tipo fenomenológico, se realizó un seguimiento durante tres años a ocho estudiantes de carreras técnicas de un centro de formación técnica que concentra una proporción relevante de la matrícula nacional, con presencia en todas las regiones de Chile, para indagar en torno a sus percepciones acerca de la efectividad del proceso de aprendizaje y a las prácticas pedagógicas utilizadas por sus docentes para el desarrollo de competencias genéricas. Los resultados sugieren que su desarrollo depende principalmente de: (a) la base de las competencias que trae el estudiante al ingresar a la institución; (b) el grado de dominio de las competencias por parte del profesor, la explicitación de los objetivos de aprendizaje y el nivel de interés que logre despertar en los estudiantes; (c) lo significativo y desafiante de las actividades diseñadas para trabajar dichas competencias; y (d) su grado de contextualización al entorno laboral.
\end{abstract}

Conceptos clave: competencia, competencia genérica, educación superior, formación técnica.

\section{DEVELOPMENT OF GENERIC SKILLS IN POST-SECONDARY VOCATIONAL EDUCATION: A CASE STUDY}

\section{ABSTRACT}

The present study aims to improve the understanding of the process of the acquisition and development of generic skills in post-secondary vocational programs, according to the students' visions and perceptions. Through a qualitative phenomenological-type case study, at the largest higher education vocational institution in Chile, eight vocational students were tracked for three years, in order to inquire about their perceptions on the effectiveness of the learning process and the pedagogical practices used by their teachers to develop generic skills. The results suggest that the development of generic skills depends mainly on: (a) the skills' foundation that the student has upon entering the institution; (b) the degree of skills' proficiency on the part of the teacher, the specification of the learning objectives and to what extent they are able to inspire student

Universidad Andrés Bello, Viña del Mar, Chile. Contacto: gerald.pugh@unab.cl Instituto Tecnológico de Sonora, Ciudad Obregón, México. Contacto: armando.lozano@ itson.edu.mx 
interest; (c) how significant and challenging the activities designed to work on generic skills are, and d) their degree of contextualization to the work environment.

Key concepts: skills, generic skills, higher education, vocational education.

\section{Introducción}

En Chile, muchas carreras técnicas de nivel superior están siendo rediseñadas bajo el enfoque de competencias, o basadas en competencias, lo que ha generado presiones sobre los modelos educativos, las estrategias pedagógicas, los sistemas de evaluación y las competencias docentes, entre otros, siendo necesario investigar cómo interactúan estos elementos para mejorar los resultados del proceso de enseñanza y aprendizaje.

Si bien los conceptos de competencia y competencia genérica se abordan en detalle en el marco teórico, es necesario anticipar que por competencia se entiende la capacidad de una persona para responder de manera efectiva a una demanda compleja en la que interactúan sus conocimientos, destrezas, valores y actitudes para abordar la tarea. Por su parte, las competencias se pueden clasificar en competencias de especialidad — propias de una determinada disciplina- y en competencias genéricas (transversales a cualquier carrera) (Lozano y Herrera, 2013). Considerando que formar en competencias supone focalizar todo el proceso en el aprendizaje del estudiante, convirtiendo al profesor en un facilitador del mismo (Villa y Poblete, 2010), se hace necesario incrementar el conocimiento que se tiene acerca de los procesos de adquisición de competencias genéricas, desde la propia perspectiva del estudiante.

La investigación se llevó a cabo en la sede Valparaíso del Centro de Formación Técnica Inacap, institución perteneciente al sistema de educación superior del país y que cuenta con una universidad, un instituto profesional y un centro de formación técnica, los que se encuentran presentes en 26 sedes distribuidas a lo largo del país, otorgando educación superior a más de 121.000 estudiantes el año 2017, equivalente al 10\% del total de los estudiantes de pregrado de Chile (Consejo Nacional de Educación, CNED, 2017). 
Los objetivos de la investigación se enmarcaron dentro de la comprensión del proceso de adquisición de competencias genéricas en estudiantes de carreras técnicas de nivel superior en la institución, principalmente, a través de la visión y percepción de los estudiantes. En este sentido, fue necesario indagar en torno a las concepciones previas que traían los alumnos acerca de las competencias genéricas al momento de incorporarse a la educación superior, el proceso de desarrollo de estas durante su formación académica, y el grado de valoración y reconocimiento en el entorno laboral, con el fin de identificar prácticas pedagógicas más efectivas, o experiencias que resulten en aprendizajes significativos, permitiendo sugerir estrategias para su desarrollo temprano en la educación superior.

El artículo se organiza de la siguiente forma. En la sección de antecedentes, se describe el contexto general sobre el cual se realiza la investigación relativo a la formación técnica de nivel superior, tanto en el ámbito nacional como internacional. A continuación, el marco teórico presenta un breve resumen de autores que han realizado aportes en el ámbito del aprendizaje basado en competencias, junto con resultados de diversas experiencias empíricas relativas al desarrollo de competencias genéricas. Luego, se presenta la metodología utilizada en la investigación, incluyendo su diseño, los instrumentos de recolección de información y la estrategia de análisis de datos. Posteriormente, se presentan los resultados organizados cronológicamente, seguido del análisis interpretativo, para finalizar con la discusión y conclusiones de la investigación.

\section{Antecedentes}

El rol de la educación para impactar en el desarrollo y bienestar de la población ha exigido cambios en la práctica educativa, con el fin de dar respuesta a una nueva sociedad globalizada y multicultural, en donde cobra una mayor importancia el desarrollo de habilidades de aprendizaje continuo y la valoración de las actividades integradoras de la teoría con la práctica (Tejada, 2000).

Esta postura pedagógica, conocida en la actualidad como "enfoque basado en competencias", desde mediados del siglo XX 
ha tenido un fuerte impacto en el diseño y ejecución de programas de capacitación para la fuerza laboral y en el diseño y provisión de carreras universitarias desde finales del mismo siglo (Lozano y Herrera, 2013).

Dentro de las diferentes modalidades de educación superior, la educación técnica de nivel superior, también conocida como "vocacional", ocupa un lugar relevante debido a su alta cobertura a nivel internacional, y a su vinculación directa con las necesidades de formación para el trabajo, ofreciendo de manera temprana oportunidades laborales a sus egresados. Sin embargo, este tipo de educación presenta fuertes debilidades estructurales en numerosos países de América Latina, las que se traducen en insuficientes recursos destinados a las instituciones formadoras y en egresados con competencias insuficientes para desempeñarse adecuadamente en el mercado laboral (Instituto de Transferencia de Tecnologías Apropiadas para Sectores Marginales, ITACAB, 2010).

Por otra parte, la creación del Espacio Europeo de Educación Superior (EEES), ha impulsado diversas iniciativas tendientes a identificar y definir las competencias necesarias, tanto de especialidad como genéricas, para las diferentes carreras universitarias, así como su nivel de importancia para el mundo del trabajo. En particular, los proyectos Tuning y Definición y Selección de Competencias, DeSeCo, han tenido un impacto sustantivo sobre las definiciones, valoración e impulso que ha tenido recientemente la formación basada en competencias (Moreno, 2010).

Bajo una mirada local, en agosto del 2015, la Comisión Nacional de Acreditación (CNA), organismo de carácter público y autónomo, que tiene como misión verificar y promover la calidad de las instituciones de educación superior en Chile, aprobó los nuevos criterios de evaluación para la acreditación de carreras profesionales, carreras profesionales con licenciatura y programas de licenciatura, en donde establece explícitamente que los planes de estudio deben incluir "el desarrollo de competencias transversales o genéricas, tales como: comunicación oral y escrita, pensamiento crítico, solución de problemas, desarrollo de relaciones interpersonales, 
autoaprendizaje e iniciativa personal, trabajo en equipo y uso de tecnologías de información" (CNA, 2015, p. 6). De igual forma, postula que la unidad que imparte la carrera debe incluir en ella "actividades formativas que promuevan el comportamiento ético, la responsabilidad social e individual, la construcción de ciudadanía y la democracia, en un marco de inclusión, de respeto a la diversidad, a los derechos humanos y al medio ambiente" (CNA, 2015, p. 6). Sin embargo, estas definiciones no dan cuenta de los propósitos esperados para la educación técnica de nivel superior.

Según las cifras oficiales de matrícula de pregrado de Chile, en el período 2005-2017 se ha producido un aumento de $82 \%$ en los estudiantes que cursan estudios superiores de pregrado. Este fenómeno se ha visto influido fuertemente por quienes han optado por una carrera técnica, quienes aumentaron 2,6 veces en igual período, alcanzando los 347.356 alumnos en 2017 , lo que representa el 30\% del total de la matrícula de pregrado, versus el $21 \%$ en el año 2005 (CNED, 2017). Por otro lado, este aumento ha provenido principalmente de estudiantes que egresan de la educación secundaria pública, a la que asisten los hijos de las familias con menos recursos económicos, y donde se reconocen falencias en el desarrollo de competencias genéricas (OCDE, 2009). A lo anterior se le suma la corta duración de las carreras técnicas las cuales, en promedio, bordean los dos años, tiempo en el que se deberán desarrollar y reforzar competencias tanto específicas como genéricas, lo que le otorga una mayor importancia a una investigación dirigida a este sector estudiantil.

El año 2011 Inacap adoptó un modelo de formación basado en competencias, lo que se tradujo en la actualización de todos sus planes de estudio, el cual se fue implementando en forma progresiva desde el 2012 al 2015. En este modelo, junto con la definición de las competencias de especialidad de cada carrera, se seleccionaron tres competencias genéricas que todo alumno tenía que desarrollar, definidas como "competencias del sello del alumno" (compromiso, autogestión y capacidad emprendedora), junto con otras siete competencias genéricas, entre las cuales cada programa de estudio debía seleccionar y desarrollar al menos tres de ellas durante la carrera 
(comunicación oral y escrita, resolución de problemas, uso de TIC, trabajo en equipo, pensamiento crítico, pensamiento creativo o ética profesional), con el fin de facilitar el ingreso y progresión en el mundo laboral de los estudiantes.

\section{Marco teórico}

Los fuertes cambios políticos, económicos, tecnológicos, culturales y sociales que ha experimentado la sociedad en las últimas décadas han generado cuestionamientos respecto del rol de la educación para asegurar el crecimiento de los países, el bienestar de sus habitantes y el desarrollo de las personas. En este contexto, Tejada (2000) destaca que "educarse hoy exige adaptarse cultural, social, laboral, profesional y personalmente al ritmo del cambio y su velocidad" (p. 1), de acuerdo con las nuevas concepciones de la sociedad, en donde cobra importancia aprender a aprender y enseñar a seleccionar y discriminar entre la información disponible. Es decir, postula que en las aulas se debe transitar desde una lógica funcionalista de la educación, basada en conocimientos, hacia el desarrollo de "competencias de concepción y acción" a través de la integración de habilidades metodológicas, técnicas y sociales, organizadas en instancias de aprendizaje que aúnen la teoría con la práctica.

Coincidiendo con la necesidad de llevar a cabo un profundo cambio en la educación, Frade (2009) lo justifica basándose en numerosas razones, entre las que destacan:

- el cambio en el estilo de aprendizaje de niños y jóvenes producto del impacto de los medios digitales de comunicación;

- el surgimiento de una sociedad de información que deja expuesta una gran cantidad de información sin análisis y sin una apropiación consciente del conocimiento;

- las transformaciones que genera la globalización, modificando los patrones y hábitos culturales de una comunidad;

- la ineficacia de los sistemas educativos en el desarrollo de habilidades de pensamiento que permitan a los estudiantes resolver problemas cotidianos; y 
- los avances en la psicología educativa y en neurociencias, que permiten comprender el funcionamiento complejo del cerebro en los procesos de aprendizaje y en su interacción con el medioambiente.

Todos estos aspectos dan cuenta de la necesidad de diseñar procesos educativos holísticos, que respondan a nuevos perfiles de egreso, de la nueva persona que se desea formar

en los que se busque integrar el mundo actual al aprendizaje (...) mediante la inclusión de conocimientos, pero también de habilidades de pensamiento, destrezas, actitudes, valores, tradiciones, costumbres y nuevas formas de relación que respondan a los retos que enfrentan (Frade, 2009, p. 71).

\subsection{El concepto de competencia}

Si bien existen diversas posturas en cuanto al origen del concepto de competencia, al igual que numerosas definiciones, es posible destacar un fuerte impulso en los últimos cincuenta años, motivado por la necesidad de contar con trabajadores más productivos y eficientes en una sociedad industrializada, el que derivó posteriormente en su incorporación en los planes y programas de estudio en educación superior, con el fin de brindar mejores oportunidades de empleabilidad a sus egresados (Lozano y Herrera, 2013).

Analizando numerosas definiciones de competencia, se distinguen tres patrones comunes en ellas:

- son una capacidad cognitivo-conductual, que se traduce en un desempeño,

- son una respuesta adaptativa a las demandas del entorno,

- incluyen conocimientos, capacidades, aptitudes, habilidades, destrezas, creencias, intuiciones, sensibilidad y motivación, lo que hace complejo su desarrollo y evaluación (Frade, 2009).

De acuerdo con la literatura de las últimas décadas, es posible identificar dos ejes de análisis, desarrollo y evaluación de las competencias. Por una parte, su aplicación en el ámbito laboral, 
con el fin de mejorar el desempeño de los trabajadores a través de la capacitación y apoyar sus planes de desarrollo de carrera (Alles, 2009; Blas, 2007; Jacinto y Millenar, 2012; Le Botef, 2001; Savaneviciene, Rutelione \& Ciutiene, 2014) y, por otra, su desarrollo en el ámbito de la educación, principalmente superior (Bennett, Dunne \& Carré, 1999; Frade, 2009; Salas, 2014; Tobón, 2009), orientado a satisfacer las demandas de las empresas y de la sociedad, desde el punto de vista de la docencia universitaria.

\subsection{Estudios derivados de las necesidades del mercado laboral}

La necesidad de las empresas de aumentar su productividad y mejorar su eficiencia ha motivado diversos estudios tendientes a identificar las competencias requeridas por sus trabajadores en los distintos puestos en los que se desempeñan.

Algunas iniciativas destacables — dada su magnitud y cobertura geográfica - apuntan a identificar las brechas existentes entre las competencias requeridas por las empresas y aquellas que forman las instituciones de educación superior, tomando en cuenta las opiniones de empleadores, de egresados y de docentes. En este sentido, el proyecto CHEERS (Careers after Higher Education: A European Research Study), conducido entre los años 1998 y 2000, marcó un hito al ser uno de los primeros estudios multinacionales en levantar información referente a la relación entre el empleo y la educación superior, obtenida directamente de graduados de 12 países, cuatro años después de su egreso. Esto permitió identificar aquellos factores que inciden en el desarrollo laboral de los graduados, tales como los niveles educacionales, la demanda de competencias especializadas o generales, la movilidad laboral internacional y la educación a lo largo de toda la vida (Teichler, 2000).

Últimamente, se han venido realizando investigaciones a nivel de cargos o posiciones laborales específicas, con el fin de identificar las competencias transversales más relevantes para ellos y evaluar su nivel de desarrollo en la educación superior. Por ejemplo, se ha identificado que los gerentes de empresas europeas de menor tamaño 
requieren principalmente competencias relacionadas con la gestión de procesos y con autogestión y relacionamiento, sin que estas sean abordadas en forma efectiva en las universidades (Savaneviciene et al., 2014).

2.3. Iniciativas que surgen desde el ámbito de la educación superior

Probablemente, el mayor impulso acerca de la valoración del concepto de competencias en la educación superior se produjo tras la creación del Espacio Europeo de Educación Superior (EEES), lo que trajo consigo nuevos desafíos a los sistemas educativos de los países europeos y un sistema de reconocimiento de grados y títulos que permitiera la movilidad de los egresados entre los países de la comunidad. En la reunión de ministros europeos de educación realizada en la ciudad de Bolonia, Italia, en el año 1999, los personeros firmaron una declaración conjunta proporcionando un marco de referencia para consolidar el EEES, de manera de avanzar hacia la convergencia de sus grados y títulos (EEES, 1999).

En este contexto, un grupo de universidades europeas propuso una respuesta concreta para posibilitar el acuerdo de Bolonia, conocido como el proyecto Tuning, el cual se caracteriza por explicitar los resultados de aprendizaje y las competencias de cada uno de los programas de estudio que se imparten en estas universidades. En dicho estudio, los resultados del aprendizaje se definieron como las "manifestaciones de lo que se espera que un estudiante sepa, entienda y sea capaz de demostrar una vez concluido el aprendizaje" (González y Wagenaar, 2006, p. 9), los cuales se expresarían en niveles de competencia a lograr, mientras que las competencias representarían "una combinación dinámica de las capacidades cognitivas y metacognitivas, de conocimiento y entendimiento, interpersonales, intelectuales y prácticas, así como de los valores éticos" (p. 9). Así, el proyecto definió una metodología para diseñar, desarrollar, aplicar y evaluar los diferentes programas de estudio en las universidades participantes, con el fin de disponer de resultados de aprendizaje comparables entre ellos. El objetivo central de la educación superior quedaría, entonces, supeditado al desarrollo de competencias, las que 
fueron divididas en competencias específicas de cada área disciplinar y competencias genéricas o transversales, comunes a cualquier programa de estudio.

En la actualidad existen diversas iniciativas como Tuning conducidas en territorios tales como Latinoamérica, África, Japón, Estados Unidos y Rusia, entre otros. Todas estas acciones mantienen las definiciones y objetivos del proyecto original, realizando solamente ajustes locales de acuerdo con la realidad cultural de cada territorio.

En el proyecto Tuning latinoamericano participaron 190 universidades de 19 países, siendo concebido como "un espacio de reflexión de actores comprometidos con la educación superior que, a través de la búsqueda de consensos, contribuye para avanzar en el desarrollo de titulaciones fácilmente comparables y comprensibles, de forma articulada, en América Latina" (Beneitone et al., 2007, p. 13). Si bien la participación de los países ha sido voluntaria y las recomendaciones del estudio no han sido vinculantes con la legislación y los modelos educativos de cada nación, actualmente son muchas las instituciones de educación superior de la región que están orientando sus perfiles de egreso y sus programas de estudio al enfoque por competencias.

\subsection{Las competencias genéricas}

Diversos autores (Beneitone et al., 2007; Corominas, 2001; Frade, 2009; González y Wagenaar, 2006; Le Boterf, 2001; Salganik, Rychen, Moser y Konstant, 1999; Tobón, 2009) han definido una primera clasificación para las competencias, en la que distinguen las competencias disciplinares o de la especialidad, aplicables a un campo formativo o área del conocimiento en particular, de aquellas competencias genéricas, de carácter general, o transversales a cualquier carrera. De esta clasificación surge una serie de términos relacionados o equivalentes, según el autor que los presente, para definir aquellas competencias que no se consideran propias de una especialidad, las que incluyen términos tales como: competencias clave, competencias transversales, competencias genéricas o competencias de empleabilidad, entre otras. Lo mismo se observa 
en la literatura en inglés, en la que coexisten los términos skills y competencies, asociados a los conceptos de key, generic, o essential, para identificar aquellos atributos o características personales, que no son propios de alguna especialidad o disciplina en particular (Young \& Chapman, 2010).

\subsection{Las competencias genéricas en el marco del proyecto Tuning}

Uno de los aportes del proyecto Tuning es la relevancia que se le ha otorgado a las competencias genéricas, su identificación, definición y clasificación, dando origen a un listado de 30 competencias genéricas en el proyecto original (Tuning Europa), las cuales fueron distribuidas en tres categorías: competencias instrumentales, competencias interpersonales y competencias sistémicas (González y Wagenaar, 2006).

En Latinoamérica, los equipos de trabajo de cada país identificaron las competencias genéricas que consideraban fundamentales, tomando como base las competencias identificadas por Tuning Europa e incorporando sus propios elementos locales y socioculturales. El trabajo consolidado dio como resultado 27 competencias genéricas, definidas como aquellas competencias compartidas "que pueden generarse en cualquier titulación y que son consideradas importantes por ciertos grupos sociales" (Beneitone et al., 2007, p. 15), las cuales se presentan en la Tabla 1. El informe concluye recomendando una enseñanza basada en competencias, con el fin de responder a las crecientes demandas de una sociedad de aprendizaje permanente y a la búsqueda de mayores niveles de empleabilidad de sus graduados. 
Tabla 1

Competencias genéricas según proyecto Tuning Latinoamérica

Capacidad de abstracción, análisis y síntesis

Capacidad de aplicar los conocimientos en la práctica

Capacidad para organizar y planificar el tiempo

Conocimientos sobre el área de estudio y la profesión

Responsabilidad social y compromiso ciudadano

Capacidad de comunicación oral y escrita

Capacidad de comunicación en un segundo idioma

Habilidades en el uso de las tecnologías de la información y de la comunicación

Capacidad de investigación

Capacidad de aprender y actualizarse permanentemente

Habilidades para buscar, procesar y analizar información procedente de fuentes diversas

Capacidad crítica y autocrítica

Capacidad para actuar en nuevas situaciones

Capacidad creativa

Capacidad para identificar, plantear y resolver problemas

Capacidad para tomar decisiones

Capacidad de trabajo en equipo

Habilidades interpersonales

Capacidad de motivar y conducir hacia metas comunes

Compromiso con la preservación del medio ambiente

Compromiso con su medio sociocultural

Valoración y respeto por la diversidad y multiculturalidad

Habilidad para trabajar en contextos internacionales

Habilidad para trabajar en forma autónoma

Capacidad para formular y gestionar proyectos

Compromiso ético

Compromiso con la calidad

Fuente: Elaboración propia sobre la base de Beneitone et al. (2007).

\subsection{Las competencias genéricas en el mundo anglosajón}

En los países anglosajones (Gran Bretaña, Estados Unidos, Canadá, Australia y Nueva Zelandia) se han realizado grandes esfuerzos para identificar las competencias genéricas necesarias para que las personas sean exitosas en diferentes contextos laborales, dando lugar 
a marcos de referencia con grandes similitudes, pero con diferencias locales. Estos países han reconocido que, desde una perspectiva económica, el desarrollo de las competencias genéricas aumenta la competitividad, eficiencia y productividad de la fuerza laboral del país, proporcionando una ventaja competitiva a nivel internacional (Lozano y Herrera, 2013).

En estos países, los marcos curriculares nacionales han considerado la importancia del desarrollo de competencias genéricas que deben formarse en los estudiantes para favorecer su inserción al mundo laboral (National Centre for Vocational Education Research, NCVER, 2003; New Zealand Ministry of Education, 2007; Qualifications and Curriculum Development Agency, QCDA, 2004; United States Department of Labor, 1991; Young \& Chapman, 2010).

A modo de referencia, en Australia, en un trabajo conjunto entre representantes del sector productivo y del mundo académico, se identificaron las competencias genéricas esenciales para las personas que se incorporaban al mercado laboral, dando origen al modelo de la ACCI/BCA (Australian Chamber of Commerce/Business Council of Australia), el que integra tanto competencias genéricas de empleabilidad como atributos personales (Tabla 2). 
156 EL DESARROLLO DE COMPETENCIAS GENÉRICAS EN LA EDUCACIÓN TÉCNICA DE NIVEL SUPERIOR: UN ESTUDIO DE CASO - G. Pugh y A. Lozano-Rodríguez

Tabla 2

Competencias genéricas de empleabilidad en Australia ACCI/BCA

\begin{tabular}{ll}
\hline Tipo & Descripción \\
\hline & Comunicación \\
& Trabajo en equipo \\
& Resolución de problemas \\
Competencias de & Iniciativa y emprendimiento \\
empleabilidad & Planificación y organización \\
& Autogestión \\
& Capacidad de aprendizaje \\
& Uso de la tecnología \\
\hline & Lealtad \\
& Compromiso \\
& Honestidad e integridad \\
& Entusiasmo \\
& Confiabilidad \\
& Equilibrio entre trabajo y hogar \\
& Motivación \\
& Presentación personal \\
& Sentido común \\
Atributos personales & Autoestima positiva \\
& Sentido del humor \\
& Capacidad para trabajar bajo presión \\
& Adaptabilidad \\
\hline
\end{tabular}

Fuente: Elaboración propia sobre la base de NCVER (2003).

\subsection{Otras aproximaciones al concepto de competencias genéricas}

Si bien existe una profunda influencia del proyecto Tuning sobre las definiciones de competencias genéricas y específicas, hay otras iniciativas que han identificado competencias que, si bien podrían catalogarse como genéricas según el marco definido por Tuning, han recibido otras denominaciones, intentando dar cuenta de concepciones más amplias, tales como las "Competencias clave para el bienestar personal, social y económico" del proyecto sobre competencias en el contexto de la OCDE, conocido como DeSeCo (Salganik et al., 1999), o las "Competencias clave para el aprendizaje permanente" recomendadas por el Parlamento Europeo y el Consejo de la Unión Europea como marco de referencia para la educación y formación de todos los jóvenes, con el fin de prepararlos para la vida adulta, las que son definidas como "una combinación de conocimientos, capacidades y actitudes adecuadas al contexto" (Parlamento Europeo, 2006, p. 13). 
De igual forma, el proyecto NML (New Millennium Learners) de la OCDE fue realizado con el fin de orientar a investigadores, educadores y legisladores en el diseño de políticas y prácticas educacionales para las nuevas generaciones de estudiantes, a la luz de los requerimientos de una sociedad del conocimiento. En él, se incluyen las llamadas "habilidades y competencias del siglo XXI", necesarias en la nueva sociedad del conocimiento (Ananiadou \& Claro, 2009), las que se agrupan en tres dimensiones: información, comunicación y ética e impacto social.

En todos estos casos resulta evidente la actual tendencia de establecer nuevos patrones conductuales, que orienten la educación y la capacitación a las nuevas necesidades de una sociedad del conocimiento, o sociedad digital, poniendo énfasis en el desarrollo de competencias genéricas.

\subsection{Competencias genéricas en la formación técnica}

Si bien los modelos de competencias mencionados responden a las necesidades de cualquier tipo de formación, la mayor parte de las investigaciones se han conducido con estudiantes universitarios, de carreras profesionales de al menos cuatro años de duración, tiempo en el cual es posible integrar en el currículo un mayor número de competencias genéricas, o bien, un mayor nivel de desarrollo de estas, lo que no suele ocurrir en la formación técnica de nivel superior, debido a la menor duración de sus programas de estudio.

\subsection{Desarrollo y evaluación de competencias genéricas en el aula}

Estudios recientes presentan la implementación de modelos curriculares que han incorporado el desarrollo y evaluación de competencias genéricas en la educación superior. En este sentido, es destacable el trabajo que realizó la Universidad de Deusto (UD), al ofrecer una propuesta para la evaluación de las competencias genéricas, basado en el proyecto Tuning, en lo que se ha denominado el Aprendizaje Basado en Competencias, ABC (Villa y Poblete, 2010). "El ABC es un enfoque de enseñanza-aprendizaje que requiere necesariamente partir de un perfil académico-profesional que recoja 
los conocimientos y competencias que se desea desarrollen los estudiantes que estén realizando un determinado tipo de estudios" (Villa y Poblete, 2010, p. 30), por lo que sugieren seleccionar un número reducido de competencias genéricas requeridas por cada carrera, de manera de incorporarlas en su malla curricular. El trabajo dirigido por estos autores presenta definiciones concretas y contextualizadas de cada competencia genérica definida por la UD, basadas en las del proyecto Tuning e incrementadas según su propia experiencia, totalizando 35 de ellas. El trabajo distingue tres niveles de desarrollo para cada competencia, desde su dominio en un contexto habitual para la persona (primer nivel), pasando por la demostración del buen uso de técnicas y formas propias del actuar competente (segundo nivel), hasta su uso en situaciones complejas, generalmente de índole profesional (tercer nivel). La extensión y profundidad de este trabajo ha permitido su uso como guía de referencia en aquellas instituciones de educación superior que están incursionando en la implementación de un modelo curricular basado en competencias.

Según Tobón (2009), las estrategias didácticas utilizadas para formar competencias se deben diseñar e implementar considerando ciertos criterios de desempeño esperado, su rango de aplicación y los tipos de evidencias requeridos para dar cuenta de los logros de aprendizaje. Entre las estrategias docentes más importantes para la formación basada en competencias destaca la estrategia de sensibilización, que favorece la atención, la cooperación, la actuación o la valoración, y otras que favorecen la adquisición, personalización, transferencia y recuperación de la información.

Si bien son numerosas las estrategias que se pueden utilizar para facilitar el desarrollo de competencias genéricas en el aula, profesores en Australia han identificado algunas que les han proporcionado buenos resultados, entre las que destacan:

- explicitar dichas competencias en el perfil de egreso,

- promover explícitamente su importancia entre los estudiantes,

- diseñar módulos separados para trabajar el desarrollo de competencias genéricas, 
- explicarlas y evaluarlas de manera más innovadora, e

- identificar profesores con un alto dominio para desarrollar estas competencias de forma que puedan apoyar a otros profesores (Callan, 2003).

Por otro lado, la formación de competencias genéricas demanda una evaluación diferenciada que permita dar cuenta de su nivel desarrollo luego de cada ciclo formativo. Para tales efectos se deberían diseñar instrumentos que dieran cuenta de las tres dimensiones constitutivas de una competencia: el aprendizaje de los saberes, el aprendizaje del actuar, y las actitudes demostradas por el estudiante (Medina, Sánchez y Pérez, 2012). La evidencia empírica sugiere que los esfuerzos se concentren en trabajar un número limitado de competencias, consideradas como más importantes para cada carrera, las cuales se puedan desarrollar a través de asignaturas específicas, o bien, integradas de forma gradual y progresiva a lo largo del programa de estudio (Corominas, 2001; Freire, Teijeiro y Pais, 2011; Palmer, Montaño y Palou, 2009; Quintana, Raccoursier, Sánchez, Sidler y Toirkens, 2007; Torra et al., 2010).

La selección de una adecuada metodología de enseñanza es fundamental para el logro de los aprendizajes esperados, debiendo ser coherente con la competencia genérica que se desea desarrollar. Una vez definidas las competencias genéricas que abordará el programa de estudio, se deberán diseñar actividades y evaluaciones que potencien su desarrollo. Por ejemplo, al evaluar diversas estrategias de enseñanza y aprendizaje, se ha observado que todas ellas tienen una contribución positiva sobre la competencia de comunicación efectiva, siendo la asignación de tareas en grupo la más efectiva para su desarrollo, en donde se privilegie la discusión de casos por sobre la lectura de textos (Salas, 2014).

\section{Metodología}

\subsection{Enfoque metodológico}

Considerando la pregunta principal de investigación acerca de cómo perciben los estudiantes de carreras técnicas su propio desarrollo de 
competencias genéricas al momento del egreso, se decidió trabajar bajo el paradigma cualitativo, considerando un estudio de caso de tipo fenomenológico, con énfasis en la obtención de percepciones o experiencias subjetivas de los estudiantes, en línea con lo planteado por Robert Stake (Denzin \& Lincoln, 2005).

\subsection{Selección de los participantes}

La investigación se llevó a cabo en la sede Valparaíso del centro de formación técnica Inacap, cuya matrícula total a inicios del 2015 fue de aproximadamente 7.000 estudiantes, de los cuales cerca de 4.000 se encontraban matriculados en carreras técnicas de nivel superior, y de estos, unos 1.700 se matricularon en su primer semestre académico.

Con el fin de reducir el sesgo producido por la participación de estudiantes con experiencia académica o laboral previa al ingreso, frente a aquellos que se matriculaban inmediatamente de terminada su enseñanza media, se seleccionaron participantes que cumplieron los siguientes criterios de significancia:

- alumnos matriculados en el primer semestre del 2015 de alguna de las carreras técnicas normalizadas bajo el nuevo modelo curricular basado en competencias;

- alumnos provenientes de liceos de enseñanza media técnicaprofesional;

- alumnos que habían egresado de la enseñanza media el año inmediatamente anterior al ingreso a la institución;

- alumnos que presentaban un rendimiento académico escolar por sobre la media de su curso;

- alumnos que presentaban una alta motivación por estudiar su carrera y claridad sobre los atributos de esta, y

- alumnos que contaban con ayuda económica del Estado que les permitiera financiar parte de sus estudios superiores, tales como becas o créditos estatales.

Las primeras tres características de los participantes (carrera, procedencia escolar y año de egreso) están asociadas directamente 
al perfil de ingreso del alumno y a las variables estudiadas, mientras que las siguientes tres (rendimiento académico previo, vocación y ayuda financiera) fueron consideradas con el fin de aumentar la probabilidad de egreso de los estudiantes en el tiempo de duración de su carrera (dos años), de manera de poder continuar la investigación en sus primeras experiencias laborales o de práctica profesional.

La información necesaria para seleccionar los estudiantes según estos criterios queda disponible al momento de matricularse, salvo la orientación vocacional, la cual fue confirmada a través de la aplicación de un test vocacional.

El diseño del estudio consideró obtener información de todo el ciclo académico de ocho estudiantes que cumplieron con los criterios de significancia, de manera de resguardar la pérdida de información producida por el eventual retiro académico de alguno de ellos, considerando que la tasa de deserción bordea el 30\% durante el primer año para este tipo de carreras. Por otro lado, se consideró trabajar con estudiantes de cuatro carreras actualizadas bajo el nuevo diseño curricular basado en competencias: Administración de Empresas, Gastronomía Internacional, Mecánica Automotriz y Técnico en Enfermería, las que presentan una alta matrícula de alumnos nuevos, permitiendo aplicar adecuadamente los criterios de significancia.

Bajo un modelo curricular basado en competencias, la institución decidió incorporar el desarrollo de diez competencias genéricas en los programas de estudio, siendo tres de ellas comunes y obligatorias para todas las titulaciones (compromiso, autogestión, y capacidad emprendedora). Adicionalmente, la institución definió tres niveles de desarrollo posible de alcanzar para cada competencia genérica: 1 (inicial), 2 (intermedio) y 3 (avanzado), los cuales identifican objetivos de aprendizaje esperados, según lo definido para cada carrera.

Por otro lado, la institución consideró que las competencias genéricas serían abordadas de manera mixta en los programas de estudio, disponiendo de asignaturas específicas de formación general 
(tales como autogestión o comunicación efectiva), o bien, integrando su desarrollo en los contenidos de las restantes materias de la malla curricular, los cuales tributan al desarrollo de una o más competencias genéricas de la carrera. Para representar lo anterior, es posible elaborar "matrices de tributación a competencias genéricas por asignatura", las que permiten visualizar el aporte de las asignaturas al desarrollo de cada competencia genérica, durante la carrera.

\subsection{Instrumentos}

Para el levantamiento de la información se consideraron las orientaciones de Stake (2007) en torno a los mecanismos para recolectar datos, validarlos y triangularlos, y la preparación previa antes de realizar observaciones de campo o entrevistas. Utilizando las denominaciones presentadas por Valenzuela y Flores (2012), se levantaron los datos a través de:

- entrevistas semiestructuradas a los estudiantes que participaron del estudio durante toda su carrera, a sus profesores de educación superior, y a sus supervisores de prácticas laborales;

- observaciones naturalistas de los estudiantes durante actividades prácticas en aulas; $y$

- a través de la revisión de documentos relevantes para la investigación, tales como los programas detallados de cada asignatura, tanto de formación general como de especialidad, los trabajos académicos generados en las materias de formación general y las definiciones institucionales de las diferentes competencias genéricas seleccionadas por las carreras para ser implementadas en su currículo.

Para conducir las entrevistas semiestructuradas se diseñaron pautas de preguntas para cada tipo de informante clave (estudiante, profesor de asignatura de formación general, profesor de asignatura de especialidad y supervisor de práctica), las entrevistas se enfocaron a su vez en identificar la forma en que las diferentes partes perciben el progreso en el desarrollo de competencias genéricas de los estudiantes participantes del estudio. Todas las entrevistas fueron grabadas en audio para su posterior transcripción y análisis. Para el registro de 
las observaciones y para el análisis de documentos relevantes, se utilizó una bitácora, en la que se incluyeron comentarios y reflexiones personales durante todo el trabajo de campo.

\subsection{Procedimiento}

Con los resultados obtenidos de una prueba piloto, y efectuados los ajustes necesarios en la metodología, se procedió a seleccionar los ochos estudiantes que participaron en la investigación, de acuerdo con los criterios definidos previamente. Este proceso se realizó durante la semana anterior al inicio de clases (primera semana de marzo 2015). Se seleccionaron dos alumnos de cada una de las carreras de Administración de Empresas, Gastronomía Internacional, Mecánica Automotriz y Técnico en Enfermería. Todos ellos aceptaron la invitación a participar del estudio, firmando un acuerdo de confidencialidad que autorizaba la grabación de las entrevistas, permitiendo resguardar su intimidad.

La investigación se condujo durante tres años, abarcando los dos años que duran las carreras, y un año para cubrir eventuales atrasos y poder observar a los estudiantes durante sus prácticas laborales. Durante este tiempo se realizaron entrevistas en profundidad al menos tres veces por año a cada estudiante, con el fin de evaluar sus progresos en el tiempo. Las entrevistas iniciales permitieron conocer a los estudiantes e indagar en torno a sus nociones previas del tema, sus ideas preconcebidas y paradigmas, además de identificar su autopercepción del nivel de desarrollo de sus competencias genéricas antes de ingresar a la institución. Adicionalmente, se realizaron observaciones de clases y entrevistas a sus profesores de asignaturas de formación general, y a profesores de asignaturas de especialidad. Por otro lado, en dos casos fue posible entrevistar a supervisores de prácticas laborales, con estudiantes ya egresados.

\subsection{Estrategia de análisis de datos}

El proceso de análisis de los datos cualitativos se realizó con base en las etapas presentadas por Valenzuela y Flores (2012), las que consideran: 
- preparación de los datos, transcribiendo las entrevistas, notas de observaciones de campo y revisión de los documentos;

- lectura de los datos, obteniendo un sentido general del tema;

- codificación de los datos, seleccionando segmentos de textos relevantes para el estudio;

- codificación de categorías a ser utilizadas en el reporte de investigación; y

- codificación de textos para descripciones a ser utilizadas en el reporte.

El análisis interpretativo de la información levantada y el desarrollo de los temas y sus categorías, por medio de lo que Corbin y Strauss (2015) denominan "microanálisis" de los datos, implicó realizar inferencias personales, cuyo sesgo fue abordado a través de la incorporación de mecanismos de control, tales como triangulaciones y member checking ${ }^{3}$, de manera de lograr una mejor aproximación a la realidad presentada por los entrevistados.

\section{Resultados}

\subsection{Selección de estudiantes}

Un aspecto clave de la investigación fue la selección de los estudiantes, con base en los criterios de significancia mencionados anteriormente, quienes durante dos a tres años compartieron sus vivencias e impresiones en las carreras que decidieron estudiar. Con la ayuda de un psicólogo, a los estudiantes se les aplicó el test de intereses vocacionales de Holland, el cual indaga acerca de las actividades que más le interesan o motivan, identificando seis tipos de personalidades ocupacionales (realista, investigativo, artístico, social, emprendedor y convencional), a partir del nivel de interés autopercibido en cuatro tipos de tareas (datos, ideas, personas y cosas). Finalmente, ocho estudiantes de cuatro carreras técnicas, con una vocación ocupacional coherente con su carrera, fueron reflexionando y compartiendo sus percepciones acerca del desarrollo de sus competencias genéricas a lo

Técnica cualitativa consistente en compartir y validar los hallazgos con los participantes de la investigación, utilizada para proporcionar confiabilidad a la información. 
largo del estudio. La Figura 1 presenta las principales características de los estudiantes, en donde se han cambiado sus nombres con el fin de mantener el anonimato. La "situación actual" indicada en la figura corresponde a la situación de cada estudiante al final de la investigación, luego de los tres años de seguimiento.

\begin{tabular}{|c|c|c|c|}
\hline Mecánica Automotriz & $\begin{array}{c}\text { Administración de } \\
\text { Empresas }\end{array}$ & $\begin{array}{l}\text { Gastronomía } \\
\text { Internacional }\end{array}$ & $\begin{array}{l}\text { Técnico en } \\
\text { Enfermería }\end{array}$ \\
\hline $\begin{array}{l}\quad \text { Miguel } \\
\text { Edad: } 18 \text { años } \\
\text { Estudios anteriores: } \\
\text { Educación técnica } \\
\text { en mecánica } \\
\text { automotriz (misma } \\
\text { especialidad) } \\
\text { Situación familiar: } \\
\text { Vive con ambos } \\
\text { padres y hermana } \\
\text { menor } \\
\text { Conocimiento } \\
\text { laboral: Si, su tío y } \\
\text { su padre trabajan en } \\
\text { taller mecánico. } \\
\text { Situación actual: } \\
\text { Continuidad } \\
\text { de estudios a } \\
\text { Ingeniería. }\end{array}$ & \begin{tabular}{l}
\multicolumn{1}{c}{ Alicia } \\
Edad: 17 años \\
Estudios anteriores: \\
Liceo Comercial \\
en la misma \\
especialidad \\
Situación familiar: \\
Vive desde pequeña \\
con sus abuelos. \\
Ahora también con \\
su padre que salió \\
de la cárcel. \\
Conocimiento \\
laboral: Sólo \\
práctica laboral por \\
el Liceo. \\
Situación actual: \\
Titulada y \\
trabajando en la \\
especialidad.
\end{tabular} & $\begin{array}{l}\quad \text { Gabriela } \\
\text { Edad: } 18 \text { años } \\
\text { Estudios anteriores: } \\
\text { Liceo Técnico en } \\
\text { Gastronomía (misma } \\
\text { especialidad) } \\
\text { Situación familiar: } \\
\text { Vive con ambos } \\
\text { padres y dos } \\
\text { hermanos menores. } \\
\text { Conocimiento } \\
\text { laboral: Sólo la } \\
\text { práctica laboral del } \\
\text { Liceo } \\
\text { Situación actual: Por } \\
\text { accidente se retrasó, } \\
\text { debiendo cursar } \\
\text { dos semestres para } \\
\text { egresar. }\end{array}$ & \begin{tabular}{l}
\multicolumn{1}{c}{ Elena } \\
Edad: 18 años \\
Estudios anteriores: \\
Liceo Comercial, \\
carrera no \\
relacionada al área \\
de la Salud. \\
Situación familiar: \\
Vive con su madre y \\
un hermano 10 años \\
menor \\
Conocimiento \\
laboral: No. Sólo su \\
vocación de servicio. \\
Situación actual: \\
Continúa \\
estudiando \\
por reiteradas \\
reprobaciones.
\end{tabular} \\
\hline $\begin{array}{l}\quad \text { Marcelo } \\
\text { Edad: } 17 \text { años } \\
\text { Estudios anteriores: } \\
\text { Liceo Industrial en } \\
\text { carrera técnica de la } \\
\text { misma especialidad. } \\
\text { Situación familiar: } \\
\text { Padres separados. } \\
\text { Vive sólo con } \\
\text { su madre desde } \\
\text { pequeño. } \\
\text { Conocimiento } \\
\text { laboral: No, sólo los } \\
\text { estudios del liceo. } \\
\text { Situación actual: } \\
\text { Titulado y } \\
\text { trabajando en la } \\
\text { especialidad. }\end{array}$ & $\begin{array}{l}\quad \text { Arturo } \\
\text { Edad: } 18 \text { años } \\
\text { Estudios anteriores: } \\
\text { Liceo Científico } \\
\text { Humanista (no } \\
\text { técnico) } \\
\text { Situación familiar: } \\
\text { Desde pequeño ha } \\
\text { vivido en hogar de } \\
\text { menores } \\
\text { Conocimiento } \\
\text { laboral: No. } \\
\text { Situación actual: } \\
\text { Egresado de la } \\
\text { carrera, realizando } \\
\text { práctica para } \\
\text { titularse. }\end{array}$ & \begin{tabular}{l}
\multicolumn{1}{c}{ Gonzalo } \\
Edad: 18 años \\
Estudios anteriores: \\
Liceo Técnico en \\
Gastronomía (misma \\
especialidad) \\
Situación familiar: \\
Vive con ambos \\
padres y dos \\
hermanos. Toca \\
música en una \\
banda. \\
Conocimiento \\
laboral: Sólo la \\
práctica laboral del \\
Liceo. \\
Situación actual: \\
Titulado y \\
trabajando en la \\
especialidad.
\end{tabular} & $\begin{array}{l}\quad \text { Eugenia } \\
\text { Edad: } 18 \text { años } \\
\text { Estudios anteriores: } \\
\text { Liceo Técnico en } \\
\text { carrerea del área de } \\
\text { deportes y salud. } \\
\text { Situación familiar: } \\
\text { Desde los } 10 \text { años } \\
\text { vive en hogar de } \\
\text { menores. Padre en } \\
\text { la cárcel. } \\
\text { Conocimiento } \\
\text { laboral: Sólo una } \\
\text { práctica del liceo, en } \\
\text { área afín. } \\
\text { Situación actual: } \\
\text { Estudió el } 2015 \text { y } \\
\text { el 2017, pero no } \\
\text { terminó }\end{array}$ \\
\hline
\end{tabular}

Figura 1. Principales características de los estudiantes participantes en la investigación. Fuente: Elaboración propia. 


\subsection{Reuniones iniciales}

Durante las primeras semanas de clases, los estudiantes fueron entrevistados con el fin de indagar en torno a sus concepciones acerca las competencias laborales en general y sobre las competencias genéricas en particular, además de identificar las percepciones acerca de sus niveles de dominio de cada competencia genérica seleccionada por la institución, al momento de su ingreso. La Figura 2 presenta un resumen de las principales observaciones levantadas en estas entrevistas iniciales.

\begin{tabular}{|l|l|}
\hline \multicolumn{2}{|c|}{ Reuniones iniciales con estudiantes } \\
\hline $\begin{array}{l}\text { Conocimiento previo de los conceptos: } \\
\text { Conocimiento y comprensión de } \\
\text { conceptos asociados a competencias } \\
\text { es muy heterogéneo y depende de la } \\
\text { influencia de sus profesores. }\end{array}$ & $\begin{array}{l}\text { Percepciones sobre el trabajo: } \\
\text { Existe una adecuada percepción del perfil } \\
\text { profesional esperado, según especialidad. } \\
\text { Son capaces de describir atributos } \\
\text { deseables, tales como responsabilidad, } \\
\text { habilidades y destrezas. }\end{array}$ \\
\hline $\begin{array}{l}\text { Desarrollo per berson el trabajo, trabajar en equipo y } \\
\text { genéricas: }\end{array}$ \\
$\begin{array}{l}\text { En generaptarse al equipo. } \\
\text { brechas entre lo que aprendieron en } \\
\text { el Liceo y lo requerido por el mercado } \\
\text { laboral. Son conscientes, principalmente } \\
\text { en comunicación. }\end{array}$ & $\begin{array}{l}\text { Predisposición para el aprendizaje: } \\
\text { La formación técnica que recibieron en el } \\
\text { Liceo les facilita comprender y asimilar el } \\
\text { modelo de formación por competencias, } \\
\text { ya que lo ven muy coherente con una } \\
\text { formación para el futuro trabajo. }\end{array}$ \\
\hline
\end{tabular}

Figura 2. Principales observaciones levantadas en las reuniones iniciales.

Fuente: Elaboración propia.

\subsection{Transcurso de la carrera}

A medida que avanzaban en sus carreras, algunos estudiantes mostraron importantes desarrollos en sus competencias de comunicación y mejoras en sus técnicas de estudio, principalmente entre quienes tenían muy disminuidas estas habilidades, logrando tomar consciencia respecto de su importancia. Por otro lado, los estudiantes que se sentían con competencias genéricas más desarrolladas, encontraron pocos desafíos en las asignaturas de formación para la empleabilidad, ya que su nivel de dominio había sido adquirido en el hogar, a través de la formación de los padres, del hábito lector, o de la práctica de algún pasatiempo. 
De los relatos de los estudiantes se desprende que, una vez que han tomado consciencia de la necesidad de dominar cierta competencia, el aprendizaje activo, colaborativo y vinculado con el mundo laboral resulta efectivo para desarrollar dicha competencia, principalmente en aquellos estudiantes que la tenían más disminuida, pero que asumieron un alto grado de compromiso personal y perseverancia para desarrollarla. La Figura 3 presenta un resumen de las principales observaciones levantadas durante el transcurso de sus carreras:

\begin{tabular}{|c|c|}
\hline \multicolumn{2}{|c|}{ Reuniones durante el transcurso de sus carreras } \\
\hline $\begin{array}{l}\text { Explicitación por parte de los profesores: } \\
\text { Salvo excepciones, los profesores no } \\
\text { les presentan en forma explícita las } \\
\text { competencias genéricas que deberán } \\
\text { trabajar en sus asignaturas, estando éstas } \\
\text { definidas en el Descriptor de la Asignatura } \\
\text { (Syllabus) }\end{array}$ & $\begin{array}{l}\text { Materias de formación para la } \\
\text { empleabilidad: } \\
\text { Las asignaturas de Autogestión, } \\
\text { Comunicación Efectiva y Competencias } \\
\text { de Empleabilidad, entre otras, tienen } \\
\text { diferente importancia para los estudiantes, } \\
\text { en función de sus niveles de desarrollo de } \\
\text { las competencias que abordan. }\end{array}$ \\
\hline $\begin{array}{l}\text { Toma de consciencia: } \\
\text { Al tomar consciencia sobre la necesidad } \\
\text { de dominar cierta competencia, el } \\
\text { aprendizaje activo, colaborativo y } \\
\text { vinculado con el mundo laboral, } \\
\text { resulta efectivo para desarrollar dicha } \\
\text { competencia. }\end{array}$ & $\begin{array}{l}\text { Actividades más significativas: } \\
\text { Destacan los trabajos grupales de } \\
\text { investigación que debieron realizar y } \\
\text { exponer frente al curso, los informes } \\
\text { manuscritos, las actividades lúdicas, y } \\
\text { las actividades prácticas en talleres. En } \\
\text { especial, las prácticas laborales. }\end{array}$ \\
\hline $\begin{array}{l}\text { Avances observados durante el transcurso } \\
\text { - Los alumnos se expresan con mayor soltu } \\
\text { - Son capaces de definir las competencias } \\
\text { como las genéricas, y reconocen la exister } \\
\text { - Identifican las competencias más relevant } \\
\text { - Son capaces de identificar sus propios ni } \\
\text { genérica. }\end{array}$ & $\begin{array}{l}\text { de sus carreras: } \\
\text { ura, y se sienten "con más personalidad" } \\
\text { de sus carreras, tanto las de especialidad } \\
\text { ncia de niveles de desarrollo para cada una. } \\
\text { tes para su futuro entorno laboral. } \\
\text { veles de desarrollo para cada competencia }\end{array}$ \\
\hline
\end{tabular}

Figura 3. Principales observaciones levantadas durante el transcurso de las carreras. Fuente: Elaboración propia.

\subsection{Al término de la carrera}

Al concluir sus estudios técnicos, los estudiantes se sentían muy orgullosos de sus logros, en especial quienes habiendo reprobado varias asignaturas, pudieron superar las dificultades, recuperar la autoestima y aprobar finalmente toda la malla curricular. Solo dos estudiantes lograron terminar la carrera en el tiempo nominal de dos años, mientras que otros tres lo hicieron durante el año siguiente. 
En términos de su percepción acerca de la adquisición de competencias genéricas que les permitirían insertarse de mejor forma en el mundo del trabajo, algunos manifestaron importantes mejoras relacionadas con la capacidad de comunicarse, tanto en forma oral como escrita, con las habilidades de planificación y con su compromiso con la carrera. El hecho de haber participado en la investigación así como el mayor dominio de sus profesores, adquirido por los programas de capacitación y de seguimiento institucional, les permitió incorporar de mejor forma estas competencias en su vida cotidiana.

La Figura 4 presenta algunas percepciones de los estudiantes referidas a su proceso de desarrollo de competencias genéricas al terminar sus carreras.

\begin{tabular}{|c|c|c|}
\hline \multicolumn{3}{|c|}{ Percepciones de los estudiantes sobre su desarrollo de competencias genéricas } \\
\hline $\begin{array}{l}\text { Comunicación efectiva: } \\
\text { Varios estudiantes } \\
\text { manifestaron una mejora } \\
\text { en su capacidad para } \\
\text { comunicarse, tanto oral } \\
\text { como escrita, en particular } \\
\text { para expresarse en público. }\end{array}$ & $\begin{array}{l}\text { Trabajo en equipo: } \\
\text { Las diversas actividades } \\
\text { realizadas en grupo, les } \\
\text { permitieron practicar y } \\
\text { reconocer la importancia } \\
\text { del trabajo en equipo. }\end{array}$ & $\begin{array}{l}\text { Dominio de las TIC: } \\
\text { El trabajo permanente con } \\
\text { computadores les permitió } \\
\text { mejorar su dominio, en } \\
\text { especial a quienes no lo } \\
\text { usaban en forma habitual. }\end{array}$ \\
\hline $\begin{array}{l}\text { Resolución de problemas: } \\
\text { Algunos estudiantes } \\
\text { reconocieron que ciertas } \\
\text { materias les permitieron } \\
\text { mejorar su capacidad } \\
\text { de análisis para resolver } \\
\text { problemas. }\end{array}$ & $\begin{array}{l}\text { Autogestión: } \\
\text { Existe una mayor } \\
\text { consciencia en la necesidad } \\
\text { de planificar antes de } \\
\text { actuar, y de aplicar nuevas } \\
\text { técnicas de estudio. }\end{array}$ & $\begin{array}{l}\text { Desarrollo personal: } \\
\text { Todos los estudiantes } \\
\text { manifestaron que se } \\
\text { sienten más maduros y } \\
\text { responsables luego de } \\
\text { sus estudios, y con "más } \\
\text { personalidad". }\end{array}$ \\
\hline \multicolumn{3}{|c|}{ Atributos más nombrados por los estudiantes } \\
\hline
\end{tabular}

Figura 4. Principales observaciones levantadas al término de sus carreras.

Fuente: Elaboración propia.

\subsection{Percepción de los profesores}

Los profesores encuentran muy positivo que la institución haya decidido adoptar un modelo basado en competencias, en especial para las carreras técnicas, y muy importante el haber explicitado la formación de competencias genéricas en las mallas curriculares. En este sentido, cada descriptor de asignatura establece las competencias genéricas que el profesor debe trabajar con sus estudiantes, y el nivel 
de dominio que se desea alcanzar al término de esta. Además, sugiere actividades prácticas y estrategias metodológicas para su desarrollo.

No obstante, un grupo de profesores encuentra que es muy elevando el número de competencias genéricas que se desea desarrollar (10), sugiriendo concentrarse en las más relevantes para cada carrera e incorporarlas en todas sus asignaturas, y no como sucede en la actualidad donde están distribuidas en diferentes asignaturas. Por otro lado, también les dificulta disponer solo de dos años para instalarlas, sin embargo, están convencidos de que se puede instalar la capacidad para que los estudiantes las continúen desarrollando por iniciativa propia.

En relación con la instauración del modelo, todos los académicos fueron capacitados a través de un curso denominado "Desarrollo de Competencias Genéricas" el cual, si bien lo encontraron muy necesario, resultó insuficiente para implementar el modelo adecuadamente en el aula, sugiriendo un mayor trabajo práctico, así como también simulaciones y la creación de comunidades académicas para compartir las buenas prácticas.

Uno de los aspectos más críticos que manifestaron los profesores de asignaturas de formación para la empleabilidad (transversales), fue la necesidad de explicitar o intencionar la competencia que se desea generar. "Se debe explicitar a los alumnos la competencia genérica que está detrás del trabajo, para que tomen consciencia de que no es solo hacer la tarea, sino que hay una serie de competencias en continuo desarrollo", mencionaba uno de ellos.

Otro aspecto observado fue la directa relación entre las competencias de entrada de los estudiantes, y la efectividad del proceso de enseñanza y aprendizaje. Por una parte, el desarrollo de competencias genéricas previas es diverso: "hay estudiantes que vienen con un bagaje anterior, que tienen más desarrolladas estas competencias; hay otros que están en proceso de desarrollo, como también hay estudiantes que les falta asimilarlas" señalaba una docente. Por otra parte, aquellos estudiantes que tenían mayor claridad en su proyecto de vida, mostraron mayor interés por las asignaturas transversales, y obtuvieron mejores resultados de aprendizaje. 
Relacionando los estudios con la futura vida profesional, varios profesores postularon que algunas competencias solo se pueden desarrollar en el mundo laboral, como el "verdadero" trabajo en equipo, distinguiéndolo del trabajo en equipo en un entorno académico, y la ética profesional, por nombrar solo un par de ellas. Por otro lado, no todas las competencias genéricas se consideran igualmente importantes, existiendo diferentes percepciones según las áreas académicas, si bien existe consenso en que "la comunicación es probablemente la más importante, y es la que se manifiesta en forma permanente en todo ámbito, ya sea el laboral, social, personal, o académico", según lo expresado por un docente de especialidad.

Finalmente, otro de los aspectos críticos es la evaluación de estas competencias, ya que "no existirían test estandarizados o únicos para evaluar una competencia, sino que habría que realizarla a través de una batería de instrumentos que permitieran ir dando cuenta de este desarrollo", según lo explicaba una profesora. Pero, incluso disponiendo de mecanismos de evaluación, existen competencias genéricas que difícilmente pueden ser evaluadas a través de conductas observables, como el pensamiento creativo o el pensamiento crítico, y otras en donde se cuestiona si realmente podrían definirse como competencias, como el compromiso y la ética profesional.

\section{Análisis interpretativo}

Considerando los resultados observados se propone la siguiente codificación de temas emergentes, de acuerdo con la propuesta de Corbin y Strauss (2015), los que resumen las principales conclusiones de la investigación en cuatro ámbitos: elementos relativos al estudiante, al profesor, al diseño curricular y al mercado laboral.

\subsection{Elementos relativos al estudiante}

Es necesario que el estudiante tenga una clara comprensión de los conceptos y del modelo educativo para que pueda abordar con éxito el desarrollo de las competencias genéricas. Durante las entrevistas iniciales se observó un conocimiento previo muy heterogéneo, por lo que la institución y los profesores deben comenzar nivelando 
el dominio de los conceptos, explicando la importancia de cada competencia, la forma en la que se abordará en el aula y los niveles esperados en cada asignatura, de acuerdo con las definiciones institucionales.

Las competencias genéricas se desarrollan a lo largo de toda la vida, por lo que existe una fuerte influencia del entorno familiar y las experiencias de vida previamente al ingreso a la institución. De las observaciones realizadas, tanto por los estudiantes como por sus profesores, se pudo constatar que mientras menor era el desarrollo inicial de una determinada competencia, los aprendizajes o cambios conductuales fueron más notorios.

La motivación por el desarrollo de estas competencias es individual y personal, y está asociada con un fuerte deseo de superación personal, cuando se toma consciencia de su importancia para el futuro laboral. Esta observación fue realizada por los profesores de asignaturas de especialidad, cuyo rol incluye la vinculación con el mundo laboral pertinente.

De igual forma, los estudiantes reconocen la necesidad de la práctica continua de la competencia genérica, bajo la supervisión del profesor, y durante un tiempo prolongado para internalizarla. Para desarrollar estas competencias ellas tienen que ser vividas, experimentadas, en ambientes de aprendizaje activos, e idealmente contextualizadas a un entorno laboral, tal como ocurre en las prácticas laborales.

\subsection{Elementos relativos al profesor}

En las entrevistas se pudo determinar que es indispensable una capacitación profunda de los profesores en el modelo de competencias y, en especial, en las competencias genéricas que adopte la institución. Es necesario acompañar al docente durante su proceso de adopción de las competencias, creando comunidades académicas para compartir buenas prácticas, y darle retroalimentación continua sobre su práctica docente. Este aspecto también fue observado en gran parte de la literatura revisada (Callan, 2003; Corominas, 2001; Tobón, 2009; Villa y Poblete, 2010, entre otros). 
Una buena práctica, muy valorada por los estudiantes, consiste en explicitarles las competencias genéricas que trabajarán en su asignatura, reforzando el concepto al momento de realizar actividades específicas para su desarrollo. Esta observación también es destacada por Callan (2003), quien además promueve la enseñanza de los estilos de aprendizaje entre sus estudiantes, para que tomen mayor consciencia de sus propios procesos de aprendizaje.

Un elemento que surgió en forma espontánea, tanto en las entrevistas con los estudiantes como con sus profesores, fue la necesidad de que el profesor tenga altamente desarrollada la competencia genérica que trabajará con sus estudiantes, y estar convencido de su importancia. Callan (2003) profundiza en este aspecto, concluyendo que los mejores profesores deberán tener atributos personales, creencias y valores que los motiven a transformar la vida de sus estudiantes.

Al trabajar una competencia genérica los profesores deberían utilizar metodologías activas y contextualizadas al futuro campo laboral de los estudiantes. Por otro lado, existe evidencia de que ciertos métodos pedagógicos son más efectivos que otros para el logro de los aprendizajes esperados, por lo que el profesor deberá adecuar sus metodologías según la competencia genérica que desee desarrollar (Salas, 2014).

La evaluación de las competencias genéricas es un elemento crítico para el aprendizaje de los estudiantes, por lo que estas deberían ser evaluadas en todas las asignaturas, con instrumentos adecuados, que incluyan rúbricas, listas de cotejo e, idealmente, portafolios de evidencias. Esta observación es reforzada por Villa y Poblete (2010), quienes proponen un modelo de evaluación que incluye una lista de conductas observables por competencia genérica para cada uno de los tres niveles de desarrollo definidos, los cuales deben manifestar los estudiantes.

\subsection{Elementos relativos al diseño curricular}

La institución debe definir y explicitar las competencias genéricas que incluirá en sus programas de estudio, de acuerdo con su modelo 
educativo, velando por tener un número reducido de ellas. Los profesores sugirieron concentrarse en un número no superior a cinco competencias genéricas, seleccionando las más pertinentes para cada carrera, en función de las necesidades del mercado laboral. Estas se podrían trabajar a través de asignaturas específicas y/o en forma integrada en todas las asignaturas.

Se pudo observar que existe interrelación entre diferentes competencias genéricas, las cuales se pueden complementar y, por lo tanto, pueden ser trabajadas en conjunto dentro de una misma asignatura, por ejemplo, el pensamiento creativo con la resolución de problemas. Por otro lado, podrían considerarse algunas competencias en todas las asignaturas, reforzando de esta manera su importancia como, por ejemplo, el trabajo en equipo y la comunicación efectiva, aportando con un sello distintivo a los estudiantes.

\subsection{Elementos relativos al mercado laboral}

Existen diferencias respecto de las competencias genéricas más valoradas, según el tipo de industria y el cargo a desempeñar, lo que obliga a las instituciones a levantar permanentemente información desde el mercado laboral. Esto se ha observado en investigaciones realizadas con empresas, las que identifican claramente las competencias genéricas más relevantes para su quehacer (Palmer et al., 2009; Savaneviciene et al., 2014).

La experiencia laboral tiene un alto impacto en el desarrollo de competencias genéricas y, en algunos casos, es el único medio válido para desarrollarlas, por lo que los profesores recomiendan incluir prácticas laborales, idealmente durante toda la carrera y no solamente al término de esta.

A modo de síntesis, en la Figura 5 se presenta la codificación de los elementos que influyen en el desarrollo de competencias genéricas en estudiantes de carreras técnicas de nivel superior. 


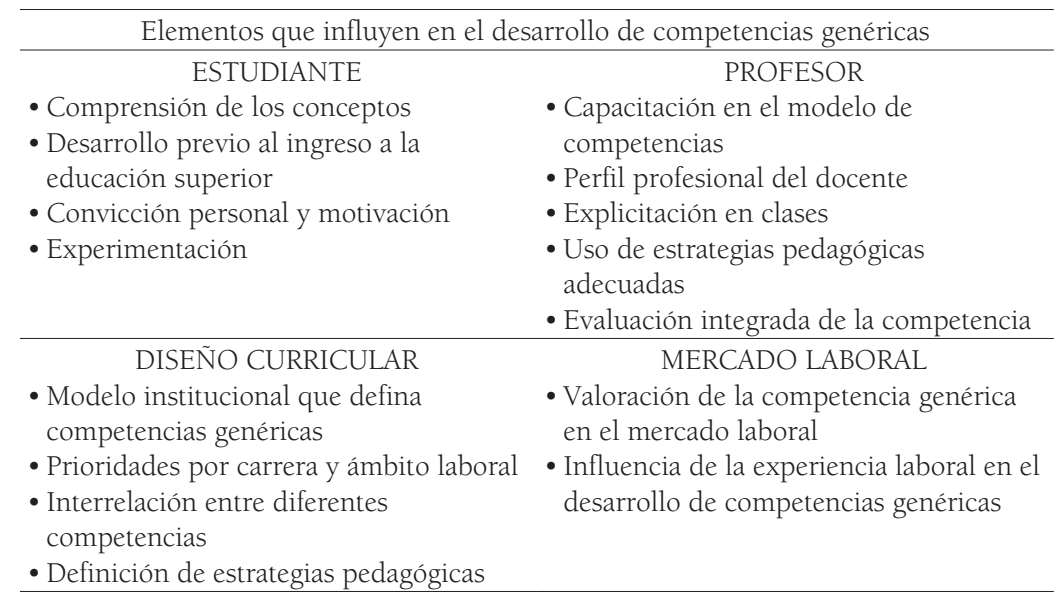

Figura 5. Principales elementos que influyen en el desarrollo de competencias genéricas. Fuente: Elaboración propia.

\section{Discusión y conclusiones}

Los resultados de la presente investigación aportan a lograr una mejor comprensión del proceso de adquisición y desarrollo de competencias genéricas en alumnos de educación superior y, en particular, de los estudiantes de carreras técnicas, de corta duración, en donde varios de los aspectos observados concuerdan con estudios conducidos en otras instituciones de educación superior.

En relación con la necesidad de disponer de definiciones claras y detalladas acerca de lo que se espera que un estudiante sea capaz de demostrar, para diferentes niveles de dominio de una competencia, ello es consecuente con el trabajo de Villa y Poblete (2010), quienes además proponen un instrumento para su evaluación.

La sugerencia de los profesores de reducir el número de competencias genéricas a trabajar con los estudiantes, limitándolas a las más importantes para cada carrera, de acuerdo con las necesidades de los futuros empleadores, es consistente con la experiencia empírica al respecto, en donde también se valida que estas se pueden desarrollar a través de asignaturas específicas, o bien, integradas de manera gradual y progresiva a lo largo de currículo (Freire et al., 2011; Palmer et al., 2009). 
Por otro lado, estudios realizados en Australia concuerdan en que se logran mejores resultados de aprendizaje cuando:

- se explicitan dichas competencias en el perfil de egreso,

- se promueve explícitamente su importancia entre los estudiantes,

- se diseñan módulos separados para trabajar el desarrollo de competencias genéricas,

- se evalúan de manera innovadora, y

- se identifican profesores con un alto dominio para desarrollar estas competencias, de manera de apoyar a otros profesores (Callan, 2003).

En relación con el nuevo rol del docente y su necesidad de perfeccionamiento para incorporar el desarrollo de competencias genéricas en el aula, el estudio concuerda con Villarroel y Bruna (2014), quienes concluyen que al incorporar competencias genéricas en la educación superior, los profesores deberán actualizarse en metodologías de enseñanza y evaluación, incorporando actividades prácticas donde puedan observar el despliegue de las competencias esperadas en la asignatura, construir evaluaciones auténticas, y entregar retroalimentación en forma permanente a los estudiantes.

No obstante lo anterior, a las instituciones de educación superior les quedan numerosos desafíos pendientes, entre los que se incluyen: la revisión de las competencias genéricas que pretende desarrollar en sus estudiantes, con el fin de lograr una mayor pertinencia con las necesidades de cada carrera; la formación y el perfeccionamiento docente; y el desarrollo y perfeccionamiento de instrumentos de evaluación que permitan monitorear el grado de avance de estas competencias en los estudiantes, a lo largo de toda su carrera. Estos tres desafíos generan oportunidades para desarrollar nuevas líneas de investigación que le permitan, tanto al centro de formación técnica Inacap como a otras instituciones formadoras de técnicos, brindar una mejor preparación a sus egresados, con el fin de que puedan enfrentar su futuro laboral con mayor seguridad en sí mismos y con mejores herramientas conductuales. 
176 EL DESARROLLO DE COMPETENCIAS GENÉRICAS EN LA EDUCACIÓN TÉCNICA DE NIVEL SUPERIOR: UN ESTUDIO DE CASO - G. Pugh y A. Lozano-Rodríguez

\section{Referencias}

Alles, M. (2009). Diccionario de competencias: la trilogía. Buenos Aires: Granica.

Ananiadou, K. \& Claro, M. (2009). 21st century skills and competences for new millennium learners in OECD Countries. OECD Education Working Papers, 41. http://dx.doi.org/10.1787/218525261154

Beneitone, P., Esquetini, C., González, J., Maletá, M. M., Suifi, G., y Wagenaar, R. (Eds.). (2007). Reflexiones y perspectivas de la educación superior en América Latina: informe final Proyecto Tuning América Latina: 20042007. Recuperado de http://tuning.unideusto.org/tuningal/index. php?option=com_docman\&task=view_category\&catid=22\&Itemid $=0$ \&order $=$ dmdate_published \&ascdesc $=$ DESC

Bennett, N., Dunne, E., \& Carré, C. (1999). Patterns of core and generic skill provision in higher education. Higher Education, 37, 71-93. https://doi. org/10.1023/A:1003451727126

Blas, F. A. (2007). Competencias profesionales en la formación profesional. Madrid: Alianza.

Callan, V. (2003). Generic skills: Understanding vocational education and training teacher and student attitudes. Recuperado de http://www.voced.edu.au/ content/ngv\%3A25598

Comisión Nacional de Acreditación, CNA. (2015). Aprueba criterios de evaluación para la acreditación de carreras profesionales, carreras profesionales con licenciatura y programas de licenciatura. Resolución Exenta $N^{\circ}$ DJ-009-4. Santiago, Chile. Recuperado de https://www. cnachile.cl/Criterios\%20y\%20Procedimientos/DJ\%20009-4\%20 Criterios.pdf

Consejo Nacional de Educación, CNED. (2017). Estudiantes de pregrado de Chile. Recuperado de https://www.cned.cl/bases-de-datos

Corbin, J. \& Strauss, A. (2015). Basics of qualitative research: Techniques and procedures for developing grounded theory ( $4^{a} \mathrm{ed}$.). Thousand Oaks, CA: SAGE.

Corominas, E. (2001). Competencias genéricas en la formación universitaria. Revista de Educación, 325, 299-321. Recuperado de https://dialnet. unirioja.es/servlet/articulo?codigo=19417

Denzin, N. \& Lincoln, Y. (Eds.). (2005). Handbook of qualitative research (3a ed.). Thousand Oaks, CA: SAGE.

Espacio Europeo de Educación Superior, EEES. (1999). Declaración de Bolonia. Recuperado de http://eees.umh.es/contenidos/Documentos/ DeclaracionBolonia.pdf 
Frade, L. (2009). Desarrollo de competencias en educación: desde preescolar hasta el bachillerato (2a. ed.). México DF.: Inteligencia Educativa.

Freire, M., Teijeiro, M., y Pais, C. (2011). Políticas educativas y empleabilidad: ¿cuáles son las competencias más influyentes? Education Policy Analysis Archives/Archivos Analíticos de Políticas Educativas, 19, 1-24. Recuperado de http://www.redalyc.org/articulo.oa?id=275019735028

González, J. y Wagenaar, R. (Eds.). (2006). Una introducción a Tuning educational structures in Europe. La contribución de las universidades al proceso de Bolonia. Universidad de Deusto. Recuperado de http://www. deusto-publicaciones.es/deusto/pdfs/tuning/tuning04.pdf.

Instituto de Transferencia de Tecnologías Apropiadas para Sectores Marginales, ITACAB. (2010). Estudio prospectivo regional. Escenarios y marco de acción estratégico de la formación para el trabajo productivo competitivo al año 2020 en los países de la organización del Convenio Andrés Bello. Recuperado de http://www.itacab.org/descarga/ prospectiva.pdf.

Jacinto, C. y Millenaar, V. (2012). Los nuevos saberes para la inserción laboral: formación para el trabajo con jóvenes vulnerables en Argentina. Revista Mexicana de Investigación Educativa, 17(52), 141-166. Recuperado de http://www.scielo.org.mx/scielo.php?script=sci_arttext\&pid=S140566662012000100007 \&lng=es\&tlng=es.

Le Boterf, G. (2001). Ingeniería de las competencias. Barcelona: Gestión 2000. Lozano, A. y Herrera, J. A. (2013). Diseño de programas educativos basados en competencias. México DF.: Editorial Digital Tecnológico de Monterrey.

Medina, A., Sánchez, C., y Pérez, E. (2012). Evaluación de las competencias genéricas y profesionales de los estudiantes. Revista Innovación Educativa, 12(58), 133-150. Recuperado de http://www.redalyc.org/ articulo.oa?id=179424061008

Moreno, T. (2010). Competencias en educación: una mirada crítica. Revista Mexicana de Investigación Educativa, 15(44), 289-297. Recuperado de http://www.redalyc.org/pdf/140/14012513017.pdf

National Centre for Vocational Education Research, NCVER. (2003). Defining generic skills: At a glance. Recuperado de http://www.voced.edu.au/ content/ngv\%3A8561.

New Zealand Ministry of Education (2007). The New Zealand curriculum. Recuperado de http://nzcurriculum.tki.org.nz/The-New-ZealandCurriculum

Organización para la Cooperación y el Desarrollo Económicos, OCDE. (2009). La educación superior en Chile. Revisión de políticas nacionales de educación. París: Autor. 
Palmer, A., Montaño, J., y Palou, M. (2009). Las competencias genéricas en la educación superior. Estudio comparativo entre la opinión de empleadores y académicos. Psicothema, 21(3), 433-438. Recuperado de http://www.redalyc.org/articulo.oa?id=72711821015.

Parlamento Europeo (2006). Recomendación del Parlamento Europeo y del Consejo sobre las competencias clave para el aprendizaje permanente. Diario Oficial de la Unión Europea, 30.12.2006. Recuperado de http:// infofpe.cea.es/fpe/norm/Rec\%2018_2006.pdf.

Qualifications and Curriculum Development Agency, QCDA. (2004). Key skills: Standards and guidance. Recuperado de http://webarchive. nationalarchives.gov.uk/20110813032310/http:/www.qcda.gov.uk/ qualifications/6263.aspx

Quintana, M., Raccoursier, M., Sánchez, A., Sidler, H., y Toirkens, J. (2007). Competencias transversales para el aprendizaje en estudiantes universitarios. Revista Iberoamericana de Educación, 44(5), 1-6. Recuperado de https://rieoei.org/RIE/article/view/2213

Salas, M. (2014). Do higher education institutions make a difference in competence development? A model of competence production at university. Higher Education, 68, 503-523. https://doi.org/10.1007/ s10734-014-9725-1.

Salganik, L. H., Rychen, D. S., Moser, U., y Konstant, J. W. (2000). Definición y selección de competencias. Proyectos sobre competencias en el contexto de la OCDE. Análisis de base teórica y conceptual. Neuchâtel: OCDE.

Savaneviciene, A., Rutelione, A., \& Ciutiene, R. (2014). Crucial transversal competences in the changing environment: Case of the European SMES Managers. Economics and Management, 19(1), 100-108. http:// dx.doi.org/10.5755/j01.em.19.1.5485.

Stake, R. E. (2007). Investigación con estudio de casos. (4a ed.). Madrid: Morata.

Teichler, U. (2000). Graduate employment and work in selected European countries. European Journal of Education, 35(2), 141-156. https://doi. org/10.1111/1467-3435.00013.

Tejada, J. (2000). La educación en el marco de una sociedad global: algunos principios y nuevas exigencias. Profesorado, Revista de Currículum y Formación del Profesorado, 4(1), 1-13. Recuperado de https://www. redalyc.org/pdf/567/56751266002.pdf

Tobón, S. (2009). Formación basada en competencias: pensamiento complejo, diseño curricular y didáctica. Bogotá: ECOE.

Torra, I., Manuel de Villena, I., Martínez, M., Gallego, I., Portet, E., y Pérez, M. J. (2010). Proceso de integración y evaluación de competencias 
genéricas en la Universitat Politècnica de Catalunya. Revista de Docencia Universitaria, 8(1), 201-224. https://doi.org/10.4995/redu.2010.6226

United States Department of Labor. (1991). What work requires of schools: A SCANS report for America 2000. Recuperado de https://wdr.doleta.gov/ SCANS/whatwork/whatwork.pdf

Valenzuela, J. y Flores, M. (2012). Fundamentos de investigación educativa. Vol. 2. [e-book]. México: Editorial Digital Tecnológico de Monterrey.

Villa, A. y Poblete, M. (Eds.). (2010). Aprendizaje basado en competencias: una propuesta para la evaluación de las competencias genéricas (3a. ed.). Bilbao: Mensajero.

Villarroel, V. y Bruna, D. (2014). Reflexiones en torno a las competencias genéricas en educación superior: un desafío pendiente. Psicoperspectivas, 13(1), 22-34. Recuperado de http://www.psicoperspectivas.cl/index. php/psicoperspectivas/article/viewFile/335/310

Young, J. \& Chapman, E. (2010). Generic Competency Frameworks: A brief historical overview. Education Research \& Perspectives, 37(1), 1-24. Recuperado de http://www.erpjournal.net/wp-content/ uploads/2012/07/ERPV37-1_Young-J.-Chapman-E.-2010.-GenericCompetency-Frameworks.pdf 